\title{
POLÍTICA DE LA FICCIÓN / FICCIÓN DE LA POLÍTICA EN JACQUES RANCIÈRE
}

\section{POLITICS OF FICTION / FICTION OF POLITICS IN JACQUES RANCIÈRE}

\author{
Azucena GONZÁLEZ BLANCO \\ Universidad de Granada \\ azucena@ugr.es
}

Resumen: Este trabajo propone analizar los sentidos que la expresión política de la ficción tiene en su doble acepción en el pensamiento literario de Jacques Rancière. Para tal fin, voy a atender a dos dimensiones en las que estaría funcionando este enunciado y que considero afectan a lo que sería el núcleo fuerte de su pensamiento, es decir, al concepto de igualdad. La política de la ficción en Rancière es, al tiempo, una estética de la igualdad. De este modo, podremos responder a la pregunta que se sitúa en el fondo de este enunciado doble, y que descansa sobre la perplejidad que su concepto de igualdad despierta: ¿Qué hace, cómo funciona, en definitiva, cómo actúa su concepto de igualdad?

Palabras clave: Política de la literatura. Performatividad. Mímesis. Ficción. A priori.

Abstract: This proposal considers analyzing certain meanings that the expression politics of fiction has in its double meaning in the literary 
thought of Jacques Rancière. The aim is to draw the conceptual map of a contradictory thought, as literature itself, that escapes the logic reason / action. I will attend the two dimensions in which this statement would be working and that I consider affect what would be the strong core of his thought, that is to say, to the concept of equality. The politics of fiction in Rancière is, at the same time, an aesthetic of equality. In this way, we can answer the question that lies at the bottom of this double statement, and that rests on the perplexity that its concept of equality awakens: What does it do, how does it work, in short, how does his concept of equality act?

Key Words: Politics of literature. Performativity. Mimesis. Fiction. A priori.

\section{LA VERDAD DE LA FICCIÓN EN RANCIÈRE}

Jacques Rancière es hoy uno de los autores más importantes y controvertidos del panorama de la teoría y la estética. Sin duda, las aportaciones de Rancière al pensamiento político, literario y filosófico han tenido como resultado uno de los debates más fructíferos de los últimos años ${ }^{1}$. Y parte de la controversia que rodea a sus trabajos está relacionada con la propuesta de conceptos fundamentales en su obra, tales como partición de lo sensible, política de la literatura, petrificación de la obra, entre otros. Para comprender los conceptos citados, se hace necesario abordar uno de los pilares del pensamiento del autor: el principio de igualdad. Es allí donde su pensamiento se sustenta sobre la paradoja de la política pasiva o la política de la igualdad de la literatura.

La hipótesis de partida es que las dos dimensiones principales en las que la política de la ficción de Jacques Rancière estaría funcionando como una estética de la igualdad se sostienen sobre su revisión de los conceptos de

\footnotetext{
${ }^{1}$ A este respecto la bibliografía es ingente. A modo de ejemplo, puede consultarse González Blanco (2019).
} 
mimesis y performatividad. Por ello vamos a aproximarnos, por un lado, a la estética de la ficción, principalmente como política de la literatura. Este concepto ha sido desarrollado en algunas de sus obras tales como Política de la literatura, La palabra muda o El hilo perdido, principalmente. El concepto de política de la literatura le permite a Rancière enfrentarse a las relaciones clásicas de la literatura entre dos opuestos, a saber, la autonomía del arte y la ideologización de la literatura. Logra Rancière de este modo una revisión del concepto de mimesis aristotélico a la luz de su crítica del concepto de verdad, que es la de la verdad como aletheia.

Por otro lado, se va a atender al concepto de estética de la igualdad como política de la ficción. Es desde esta segunda acepción desde donde Rancière abordaría el problema de la pérdida de los grandes relatos del siglo pasado. En este primer avance, la estética política de Rancière se plantea como una estética pasiva, si bien en su origen encontramos una performatividad que funciona como un condicional apriorístico ${ }^{2}$, como va a argumentarse en las siguientes páginas.

Para ello, el trabajo se va a plantear y estructurar desde una metodología crítico-interpretativa. Con dicha metodología se quiere alumbrar aspectos fundamentales de la teoría política de la literatura de Rancière. Estos aspectos fundamentales van desde la revisión de las relaciones entre política y literatura tal y como se había definido en el siglo pasado, su definición de partición de lo sensible; hasta otros menos evidentes como son la revisión del concepto de aletheia en relación con el concepto de mimesis que propone en sus obras. Por último, en el horizonte de este trabajo está intentar responder al cuestionamiento desde la crítica materialista a su radical pasividad. Con ello pretendemos poner de relieve una compleja pasividad.

\footnotetext{
${ }^{2}$ Podríamos decir, para seguir con la terminología aristotélica, que sería el primer motor inmóvil de ese principio de igualdad.
} 


\section{MIMESIS, ALETHEIA Y DESAUTOMATIZACIÓN}

Rancière funda su pensamiento crítico en lo que denomina la partición de lo sensible (le partage du sensible) que define como:

Llamo reparto de lo sensible a ese sistema de evidencias sensibles que permite ver al mismo tiempo la existencia de un común y los recortes que definen sus lugares y partes respectivas. Un reparto de lo sensible fija al mismo tiempo algo común repartido y ciertas partes exclusivas (Rancière, 2014: 17).

Rancière denuncia así que la división política coincide con la imposición de "una separación de acuerdo al uso de la palabra que está determinado por el ejercicio de una u otra tekné” (34). Desde Platón, la determinación ontológico-política depende de la acción como principio racional. Esto es: no podemos pensar dos cosas al mismo tiempo porque no podemos hacer dos cosas al mismo tiempo, porque no tenemos el tiempo para hacerlas simultáneamente.

Según Rancière, estas fronteras entre los modos discursivos estarían en la base de nuestra democracia y precederían a la división entre los que gobiernan y los que son gobernados. Sobre ella se asentaría también la fundación antropológica que coincide con la partición política de lo sensible y con una estética, que es una estética política. Tal definición es la que quedaba enunciada por Aristóteles en su Política del hombre como animal político. Es decir, aquella que separaba a los que tienen derecho a hablar y los que no, entre los que tienen la palabra y los que no. Esta crítica a la fundación desigual de la democracia está muy próxima al concepto de democracia acotada que Etiènne Balibar formula en Violencias, identidades y civilidad (1997), para quien la frontera y la partición están en el origen de la democracia y, al mismo tiempo, la contradicen ${ }^{3}$. Como para

${ }^{3}$ E. Balibar se planteaba una cuestión clave para la comprensión del concepto de frontera. El trabajo de Balibar permite trazar una geneaología del término latino limes en sus 
Rancière que habla de agravio, Balibar habla de "la violencia legítima" de nuestras sociedades del norte.

Como consecuencia de esta co-fundación de la partición políticolingüística, estética y antropológica, la política va a ser definida por Rancière en estos términos: "La política se refiere a lo que vemos y a lo que podemos decir, a quien tiene la competencia para ver y la cualidad para decir, a las propiedades de los espacios y los posibles del tiempo" (2014: 20).

Pero aquí debemos plantearnos otra cuestión, ¿cómo se relaciona el arte y la literatura con la política desde esta perspectiva y en relación con la tradición aristotélico-platónica? No olvidemos que la poiesis en la época clásica estaba contemplada como una categoría de la tekné. Por lo que el arte, en su fundación premoderna, participa de esta separación. Por ello, la estética que está en la base de la política determina que las prácticas artísticas participen de lo común en tanto que maneras de hacer que intervienen en la distribución general de las formas de ser y las formas de visibilidad (2014: 20). Por ello, según Rancière, el problema de la literatura, el motivo por el que expulsa Platón a los poetas es debido, precisamente, a que sitúa a los ciudadanos fuera de su lugar. Es decir, el problema de la poiesis sería, para Platón, que el teatro se funda "en la imposibilidad de hacer dos cosas al mismo tiempo" (2014: 20) y porque "confunde el reparto de las identidades, de las actividades y los espacios"

distintas acepciones a lo largo de la historia. Si bien es cierto, dice Balibar, que hay identidades (activas y pasivas, deseadas y padecidas, individuales y colectivas) en distintos grados. Y, como los muros, su multiplicidad, su carácter de construcciones o de ficciones, no las tornan menos violentas (la violencia legítima de nuestras sociedades del norte). Balibar, en definitiva, pone el acento en la relación entre identidad y frontera, y en su formulación más espectacular, entre identidad y muro: desde los Estados se constituyen los sujetos, cuyos precedentes están en los Estados nacionales-imperiales bajo la forma de súbditos. Esto pondría en evidencia que "en la complejidad histórica del concepto de frontera, que vuelve a presentarse ante nosotros y al mismo tiempo evoluciona y reviste nuevas formas, anida la problemática de la institución" (Balibar, 2005: 85). Es decir, la frontera se presenta como condición de posibilidad para una multiplicidad de instituciones que pueden ser o no democráticas, si bien el muro es, en sí mismo, antidemocrático. Es lo que Balibar llama "la democracia acotada". 
(2014: 21). Por ello Platón y Aristóteles habrían hecho coincidir una jerarquía ontológica, antropológica y política con una jerarquía estética en lo que Aristóteles llama fábula. Para Rancière, por lo tanto, la cuestión de la ficción es una cuestión de distribución de los lugares, y la literatura en la modernidad vendría a desregularizar la estructura o jerarquía de los seres políticos. Y ello porque, en opinión de Rancière, la literatura moderna es fundamentalmente democrática y descompensa las estructuras y las reglas de representación del sentido de la comunidad.

Pero ¿cómo lo hace, cómo se enfrenta a la estructuración del espacio perceptivo, aquello que Rancière llama la policía? Según expone Rancière en la entrevista que Éric Alliez le realiza en 2000, “¿Biopolítica o política?", la literatura enfrenta a la estructura el conjunto, “un conjunto de actos que efectúa una propiedad suplementaria: la igualdad de los seres hablantes. La literatura actúa entonces como artificio de la igualdad, como una ficción de la igualdad" (Rancière, 2011a: 123).

Por lo tanto, a la división que está en la base de la política, la literatura opondría el hecho transparente de la ut pictura poiesis. Pero no como un mero volver al dictamen clásico, sino muy al contrario, sustrayendo el concepto de verdad como veritas que se encuentra en la base de la sentencia horaciana, e introduciendo aquí una revisión política de la aletheia. Rancière introduce, frente a la veritas tradicional en la que coinciden la palabra y la cosa, una aletheia que desvincula aquí la verdad de la razón. Al contrario que para Platón y Aristóteles, la verdad ya no es adecuación con la idea y la percepción no será tampoco mirar la idea como en el mito platónico. Mientras que la verdad de la veritas se identificaba con la rectitud de la representación enunciativa, la aletheia funciona como un modo ficcional de emancipación en tanto que trasciende la partición política de lo sensible.

El concepto de aletheia ya fue revisada en el siglo XX por S. Freud y M. Heidegger, entre otros. Y en ambos, el concepto de Unheimlich juega un papel fundamental ${ }^{4}$. Para Freud lo siniestro, recuérdese, es un

${ }^{4}$ En este sentido también puede leerse el concepto de Lacan de la angustia, que define 
fenómeno donde lo que fue familiar se transforma en inquietante y lo que debía permanecer oculto se ha manifestado. En el caso del Heidegger de "El origen de la obra de arte" (1935), en la palabra literaria acontece (Ereignis) la verdad como aletheia adquiriendo la obra un estatuto muy próximo al del monumento en Rancière "lo que conserva la memoria por su propio ser" (2013, posic. 184 de 829):

Es verdad que el escultor usa la piedra de la misma manera que el albañil pero no la desgasta. En cierto modo esto sólo ocurre cuando la obra fracasa. También es verdad que el pintor usa la pintura, pero de tal manera que los colores no sólo no se desgastan, sino que gracias a él empiezan a lucir. También el poeta usa la palabra, pero no del modo que tienen que usarla los que hablan o escriben habitualmente desgastándola, sino de tal manera que gracias a él la palabra se torna verdaderamente palabra y asi permanece (Heidegger, 2001: 34).

El concepto de verdad en Heidegger como aletheia supone un desocultamiento de lo que es en sí el objeto. Así pues, la verdad de la bota de labrador es su utilidad para el trabajo en el campo, su llamada por la tierra. El olvido del ser es el olvido de la definición genuina del ente, es la automatización. Es por ello que, como para los formalistas, el arte es el camino para la verdad, pues la desoculta, la desautomatiza y reabre el combate entre historia y mundo, dice Heidegger "para que la lucha siga siendo lucha". Por lo tanto, la verdad de la obra de arte acontece como "darse a ver" (aletheia) mediante el extrañamiento de lo familiar (Unheimlich). La literatura extraña la lengua y nos la presenta de otro modo.

como un modo de percepción de lo real.

${ }^{5}$ Nótese, sin embargo, que la diferencia que nos asalta al comienzo del texto heideggeriano con respecto a la propuesta de Rancière, es que Heidegger deja muy claro que de lo que habla es de lo que se considera "el gran arte". Concepto que no encontramos en Rancière, que ha trabajado en igualdad de condiciones tanto literatura como cine. La igualdad de los géneros artísticos es una constante en la obra de Rancière. 
En términos de teoría literaria clásica, la función de la literatura coincidiría con el concepto de Shklovski de extrañamiento u ostranénie que conlleva la desautomatización o percepción de la alienación del lenguaje con el que nos relacionamos. Según el teórico ruso:

El propósito del arte es el de impartir la sensación de las cosas como son percibidas y no como son sabidas (o concebidas). La técnica del arte de 〈extrañar〉 a los objetos, de hacer dificiles las formas, de incrementar la dificultad y magnitud de la percepción encuentra su razón en que el proceso de percepción no es estético como un fin en si mismo y debe ser prolongado. El arte es una manera de experimentar la cualidad o esencia artística de un objeto; el objeto no es lo importante (Shklovski, 1991: 70).

Por su parte, Rancière va a definir la literatura y el arte como como el lugar en el que "acontece su propia verdad" en la indiferencia del tema y la necesidad de la obra. La literatura es, por tanto, "el puro dar a ver" en el estado de perplejidad o mudez (Unheimlich) que produce la literatura moderna como poética contradictoria, lo que el autor denomina "la letra muda" (Rancière, 2009). En esta palabra muda el concepto de escritura se desdobla y es, al mismo tiempo, palabra huérfana de todo cuerpo capaz de conducirla o atestiguarla; y jeroglifico que lleva la idea de escritura en su propio cuerpo (Rancière, 2009: 12). Y la contradicción de la literatura bien podría definirse como la tensión entre estas dos escrituras.

De este modo, el concepto de aletheia en Rancière, como este dar a ver, es una crítica profunda de la mimesis que Aristóteles define como “imitación de acciones humanas". Y está muy próxima a la definición del concepto que Auerbach describiera en su obra homónima en 1942:

En la literatura moderna, todo personaje, cualquiera que sea su carácter y posición social, y todo episodio, tanto fabuloso como de alta política, o limitado a lo doméstico, pueden ser tratados 
por el arte imitativo, y lo son la mayoría de las veces, de manera severa, problemática y trágica. En la antigüedad esto es totalmente imposible. Aunque en los poemas pastoriles y eróticos existan algunas formas intermedias, en conjunto rige la regla de la separación de estilos [...]: lo que corresponde a la realidad vulgar, a lo cotidiano, no puede ser presentado más que en la comedia, sin ahondamientos problemáticos (Auerbach, 2008: 37).

Y ello, afirma Rancière, por su falta de consciencia histórica. Piensa Rancière, entonces, con Auerbach y contra Aristóteles, que la literatura se aproxima más a la historia que a la filosofía, cuestionando el principio de verosimilitud que rige las formas clásicas de la poiesis. Para Rancière, como también para Agamben, relatar la Historia es en definitiva contar una historia:

Una historia es una disposición de acciones por la cual no solo ha habido primero esto y luego a su vez eso otro, sino también una configuración que une hechos y permite presentarlos como un todo: lo que Aristóteles llamó mythos; una trama, un argumento o fábula, en el sentido en el que se habla del argumento de una obra de teatro (Rancière, 2013: posición 64 de 829).

Pero la historia, no coincide con la propuesta aristotélica porque, para Rancière, al contrario no existe ningún lazo de causalidad que ordene la trama y legitime el orden establecido. Rancière realiza así una crítica de la naturaleza narrativa de la historia utilizando los recursos del análisis literario. Por lo que, la política del texto literario en particular, y el papel de la literatura y el arte en su relación con la historia, es reordenar la historia oficial de otro modo. Y así el arte y la literatura "afirman tranquilamente la igual aptitud de todos para todos los aprendizajes" (Rancière, 2013: posición 146 de 829). La historia, entonces, queda reordenada y "es el tiempo en el que aquellos que no tienen derecho a ocupar el mismo 
lugar pueden ocupar la misma imagen" (Rancière, 2013: posición 113 de 829). La literatura y las artes son capaces de este modo de contar de otra manera, y ello lo hacen, dice Rancière, "consciente o inconscientemente. Voluntariamente y más allá de lo que se quería"6 (Rancière, 2013: posición 146 de 829). Y ello porque la literatura permite la "suspensión entre dos regímenes de la explicación". En esta suspensión acontece la verdad como memoria o lo que Rancière describe como:

el despliegue de la plenitud plegada en su simple presencia [...] Cortando el hilo de toda razón, se deja la escena, la actitud, el rostro al mutismo que les confiere un doble poder: detener la mirada en esa evidencia de existencia ligada a la ausencia misma de razón, desplegar dicha evidencia como virtualidad de otro mundo posible (Rancière, 2013: posición 153 de 829).

Otro mundo posible que, sin embargo, ya es, sólo que no lo habíamos visto, no le habíamos otorgado presencia. La literatura actualiza, entonces, una virtualidad del presente al-alcance-de-la-mano, por utilizar la expresión con la que Agamben responde a la metafísica de la presencia (2016).

De esta manera, Rancière responde a la determinación que vinculaba acción y pensamiento racional. La virtualidad de otro presente permite así:

1. Pensar dos cosas simultáneamente, al mismo tiempo y oponerse de este modo a la determinación acción-razón que estaba en la base de la partición de lo sensible desde Platón y Aristóteles que atribuía una situación y un tiempo a cada ciudadano de acuerdo con la tekné que desarrollaba.

\footnotetext{
${ }^{6}$ Abro aquí un paréntesis para referirme a este inconscientemente: La dimensión ontológica del texto literario tal y como lo habían descrito M. Blanchot o M. Foucault en sus textos de los sesenta, quedaría aquí reinterpretada como una ontología política del texto. La dimensión lúdica de este decir sin voluntad (recordemos el texto de Foucault sobre Raymond Roussel) es reinterpretada por Rancière como el puro dar a ver del texto literario. Por ello para Rancière la naturaleza del texto literario es radicalmente política.
} 
2. Proponer un modo de pensar el tiempo de las nuevas mitologías como virtualidad del presente. Alejado ya del futuro de las utopías, y en la línea de otros trabajos que se vienen realizando desde Deleuze y más recientemente con Agamben.

Por lo tanto, la verdad de la literatura es una virtualidad del presente: a la vista de todos pero, al mismo tiempo, oculta. De este modo, Rancière devuelve a la literatura su carácter desautomatizador en tanto que contradictorio (esta era la tesis fundamental de La palabra muda) y a la aletheia un valor radicalmente político.

\section{NUEVAS MITOLOGÍAS: EL RELATO DE LA IGUALDAD}

Recuérdese que para Rancière la política de la literatura "es indisolublemente una ciencia de la sociedad y la creación de una mitología nueva" (Rancière, 2011b: 39). Llegamos así al segundo sentido en el que interpretamos esta "política de la ficción": a saber, la propuesta de una estética de la igualdad estaría funcionando como una mitología nueva en la que la igualdad se propone como a priori histórico de todo pensamiento. Porque, podríamos simplificarlo mucho, la emancipación es posible si primero pensamos que es posible. De este modo, la lógica de la igualdad queda trazada frente a la lógica de la distribución que es también la lógica del pensamiento no contradictorio (según Aristóteles, la posibilidad del pensamiento no puede escapar al principio de identidad).

El trabajo de Christoph Menke de 2013, La fuerza del arte, nos permite delimitar el alcance de este a priori histórico y lo que define esa igualdad política como una estética. En este trabajo, Menke aborda el principio de igualdad como a priori. Citando a H. Arendt, Menke considera que la afirmación en la que Arendt considera que el hombre no es libre ni igual por naturaleza, haría referencia a un estado pre-político de la naturaleza. Para el alemán, ello supone aceptar que adquirimos nuestras capacidades a través de la educación, el entrenamiento y la práctica. Por lo tanto, para Menke, la igualdad es una igualdad de la potencialidad, una 
igualdad de fuerza ( $k r a f t)$, no algo dado.

Por lo tanto, es necesario llegar a la igualdad no como algo dado, sino como una ficción. Y utilizo el término ficción en el mismo que se ha venido describiendo en este trabajo. Pero, a diferencia de Menke, la estética de la igualdad de Rancière sólo se puede formular en su radical inactividad: es necesario pensar la igualdad lejos de la lógica de la acción, porque esta ha sido la que, desde su origen, ha determinado la división de los cuerpos como escenificadores de una tekné, de unas capacidades. Como subraya Rancière: "La acción es un modo de pensamiento, una estructura de racionalidad que define, a la vez, unas normas de comportamientos sociales legítimos y una norma de composición de las ficciones" (Rancière, 2015: 100).

De este modo, la política de la ficción/la ficción de la política se define en Rancière a través de su definición angular del concepto de igualdad como un a priori. Un a priori que funciona, a la vez, como un presente virtual, por lo tanto, como un a priori histórico: un como si que consigue traer al presente una actualización de la igualdad sin caer en la lógica de la acción que es, en último lugar (o en primer lugar), la que inaugura la escisión (reparto de lo sensible). De este modo, desde esta virtualidad, lo imposible se convierte en real.

Así responde Rancière a la tiranía de la trama como verosimilitud lógica que aproximaba la verdad a la coherencia y dejaba fuera aquello posible y verdadero pero inverosímil, desde Aristóteles. Y, al mismo tiempo, el principio de igualdad es, también, un a priori performativo. La acción por el enunciado otro: el mundo se enuncia como si fuera otro. La posibilidad de esta formulación contradictoria pertenece a la naturaleza misma de la literatura, es decir, es una lógica ficcional y no racional. 


\section{REFERENCIAS BIBLIOGRÁFICAS}

AGAMBEN, G. (2016). El fuego y el relato. Madrid: Sexto Piso.

AUERBACH, E. (2008). Mimesis. La representación de la realidad en la literatura occidental. México: Fondo de Cultura Económica.

BALIBAR, E. (2005). Violencias, identidades y civilidad. Para una cultura política global. Barcelona: Gedisa.

GONZÁLEZ BLANCO, A. (2019). Literatura y politica. Nuevas perspectivas teóricas. Berlín: De Gruyter.

HEIDEGGER, M. (2001). El origen de la obra de arte. Madrid: Alianza.

MENKE, C. (2017). La fuerza del arte. Santiago de Chile: Metales Pesados. RANCIÈRE, J. (2009). La palabra muda. Ensayo sobre las contradicciones de la literatura. Buenos Aires: Eterna Cadencia. (2011a). “Biopolítica o política?”. En El tiempo de la igualdad. Diálogos sobre política y estética, 121-127. Barcelona: Herder. (2011b). Politica de la literatura. Buenos Aires: Zorzal. (2013). Figuras de la historia. Buenos Aires: Eterna Cadencia. Edición kindle.

(2014). El reparto de lo sensible. Estética y política. Buenos Aires: Prometeo.

(2015). El hilo perdido. Ensayos sobre la ficción moderna. Madrid:

Casus Belli. (2017). Les bords de la fiction- Paris: Seuil.

SHKLOVSKI, V. (1991). "El arte como artificio". En Teoría de la literatura de los formalistas rusos. Antología, Tzvetan Todorov (ed.), 55-70. México: Siglo XXI.

Recibido el 22 de abril de 2018.

Aceptado el 4 de septiembre de 2018. 
